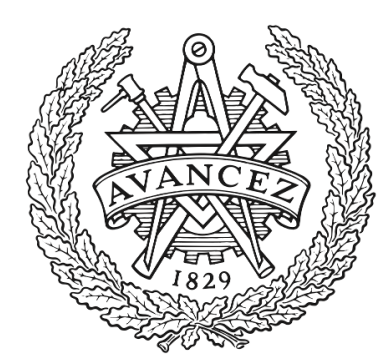

\title{
CHALMERS
}

UNIVERSITY OF TECHNOLOGY

\section{G RAN architecture based on analog radio-over-fiber fronthaul over UDWDM-PON and phased array fed reflector antennas}

Downloaded from: https://research.chalmers.se, 2023-04-26 01:24 UTC

Citation for the original published paper (version of record):

Konstantinou, D., Bressner, T., Rommel, S. et al (2020). 5G RAN architecture based on analog radio-over-fiber fronthaul over UDWDM-PON and phased array fed reflector antennas. Optics Communications, 454. http://dx.doi.org/10.1016/j.optcom.2019.124464

N.B. When citing this work, cite the original published paper. 


\title{
5G RAN Architecture based on Analog Radio-over-Fiber Fronthaul over UDWDM-PON and Phased Array Fed Reflector Antennas
}

\author{
Dimitrios Konstantinou ${ }^{\mathrm{a}}$, Thomas A. H. Bressner ${ }^{\mathrm{a}}$, Simon Rommel ${ }^{\mathrm{a}}$, \\ Ulf Johannsen $^{\mathrm{a}}$, Martin N. Johansson ${ }^{\mathrm{b}}$, Marianna V. Ivashina ${ }^{\mathrm{c}}$, \\ A. Bart Smolders ${ }^{\mathrm{a}}$, Idelfonso Tafur Monroy ${ }^{\mathrm{a}}$ \\ ${ }^{a}$ Eindhoven University of Technology, 5600MB Eindhoven, Netherlands \\ ${ }^{b}$ Ericsson $A B$, Sweden \\ ${ }^{c}$ Chalmers University of Technology, Sweden
}

\begin{abstract}
This manuscript introduces a 5G radio access network architecture concept based on ultra-dense wavelength division multiplexing (UDWDM) and incorporating an optical fronthaul network that uses a novel wireless antenna system for radio frequency transmission and reception. A ring topology is proposed where optical signals travel within the 5G UDWDM passive optical networks and millimeter waves are generated in the optical line terminals by optical heterodyning. The wireless transmission of the millimeter waves is conducted by an innovative phased array fed reflector antenna approach for mobile communications that grants high antenna gain due to highly focused radiation characteristics, as well as multiplexing gain by multiple beam generation. Furthermore, beam steering is provided by a radio frequency analog beamformer network. Finally, implementation options synthesizing the total system are discussed.
\end{abstract}

Keywords: 5G, UDWDM PON, mm-wave, reflector antennas, analog radio-over-fiber, beam steering

2010 MSC: 78A50, 78A60, 78-05, 78A25,

\section{Introduction}

The fifth generation (5G) of mobile communications will bring massive advancements to the radio access networks (RANs) and revolutionize the existing 
network infrastructure. The increasing number of mobile devices that entail demanding internet applications have boosted the need of higher capacity with enhanced coverage potential [1]. Based on ITU-T FG-IMT-2020 [2, 5G networks should provide 1000 times more wireless capacity supporting internet connection to over 7 trillion wireless devices among 7 billion people with exceptionally low latency $(<1 \mathrm{~ms})$. Especially concerning the capacity in small cells, it is expected that the data rate requirements will range between 100 to 1000 Mbps with a peak of 10 Gbps. Furthermore, mobile phones, vehicles and robotic devices have to be supported and controlled with ultra-fast connectivity by these networks [3]. In addition, $5 \mathrm{G}$ networks should be energy efficient contributing to the decrease of $\mathrm{CO}_{2}$ emissions [4. This will be achieved by focusing on the reduction of the energy consumption within the RANs.

The use of communication signals in millimeter wave (mm-wave) bands will decisively increase the capacity of future $5 \mathrm{G}$ networks. Due to the fact that in mm-wave bands a large amount of small antennas can be used, beamforming (both in transmission and reception) is one of the major technologies that is feasible to be introduced into these systems. Consequently, 5G systems can support multiple-input multiple-output (MIMO) technology, boosting even further the spectral efficiency [5]. However, at mm-wave frequencies the radio signals suffer from high path loss (atmospheric attenuation, rain fading, and foliage attenuation) [6] and reduced coverage for non-line-of-sight (NLOS) connections. One possible solution to this impediment is the size reduction of the current cells and the increase in the number of network nodes located in a specific area. Passive optical networks (PONs) [7] are candidates for being the network to support connections in small cells. Small cells have coverage areas with radius of 10-200 $\mathrm{m}$ and the antennas within radiate at power levels compensating for the high path loss 8 . Nonetheless, the current structure of PONs does not allow them to handle the vast amount of connections needed towards $5 \mathrm{G}$ because of the limited data rates and the small splitting ratios of the PONs 9 .

The EU-H2020 project-ITN 5G STEP FWD [10, promises to transform the current PONs to UDWDM PONs that are able to support a very high number 
of wavelengths available to be designated to different small cells [11. This means that the architecture uses ultra narrow spacing $(6.25 \mathrm{GHz}$ or $12.5 \mathrm{GHz})$ between wavelengths in order to assure connections within highly dense small cell networks. This is done by introducing and implementing a UDWDM PON architecture, supporting hyper dense mm-wave fronthaul networks based on 5G requirements [12]. Furthermore, during this project, the interface between the optical network unit (ONU) and the mm-wave antenna will be designed. Finally, this $5 \mathrm{G}$ architecture will use orthogonal frequency division multiplexing (OFDM) modulation as in 4G. However, 5G will support a new and flexible numerology [13].

To truly expand wireless communications into the outer limits of radio technology, the novel optical 5G STEP FWD approach will be connected to the phased array fed (PAF) reflector antenna system of the EU-H2020 project-ITN SILIKA [14. While PAF reflector antenna systems are usually applied in radio astronomy [15] and considered for space-based sensor applications [16], they are also a promising approach for 5G mobile communications. By utilizing PAF reflector systems, multiple-beam generation with highly focused beam characteristics is achievable. Furthermore, due to the focused energy, the system has the potential to reduce hardware complexity as well as signal processing requirements. Hence, by combining the optical system of 5G STEP FWD and the antenna approach of SILIKA, an improved user experience can be provided.

\section{Analog Fronthaul Radio Access Network Based on UDWDM PON}

WDM PONs are most commonly associated with fiber to the x (FTTx) applications, where $\mathrm{x}=$ home, curb, or bulding [17]. UDWDM is the next step to the evolution of optical access networks, since the wavelengths of each channel that are multiplexed within a single fiber are spaced either by $6.25 \mathrm{GHz}$ or 12.5 GHz. 5G STEP FWD proposes the use of a ring architecture interconnecting multiple UDWDM PONs, shown in Fig. 1. This structure also allows the optical line terminals (OLTs) to interchange information through optical add 


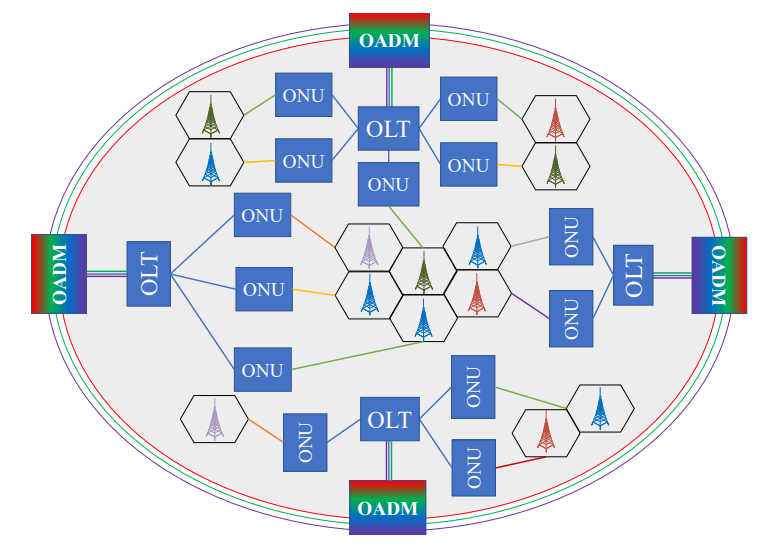

Figure 1: The ring infrastructure of the UDWDM PON based on the approach of 5G STEP FWD.

drop multiplexers (OADM).

Cost effectiveness is a major target of the project. One way to achieve this goal is by properly combining the modules (both passive and active) of the networks providing fast and reliable connections, between the PONs and the end users [18]. Moreover, the activation of the dark fibers within the existing infrastructures could support the vast amount of connections towards the evolution to $5 \mathrm{G}$.

The core of the concept that $5 \mathrm{G}$ STEP FWD proposes is that the mm-wave RoF links will be integrated with the already installed PONs and their future versions (e.g., UDWDM PONs). A basic description of the suggested network is shown in Fig. 2, Within the central office (CO) the signal generation, distribution and the control of channel interleaving is conducted. Combining analog radio over fiber (ARoF) fronthaul with flexible carrier aggregation allows optimum resource utilization. The resource management and allocation within the network are offered by a virtual orchestrator based on a centralized control plane defined by software defined networking (SDN) and network function virtualization (NFV) [19]. The centralization of the RANs leads to a more flexible routing as well as it allows the reduction of the complexity within the ONUs increasing control and minimizing the communications latency [20, 21]. 


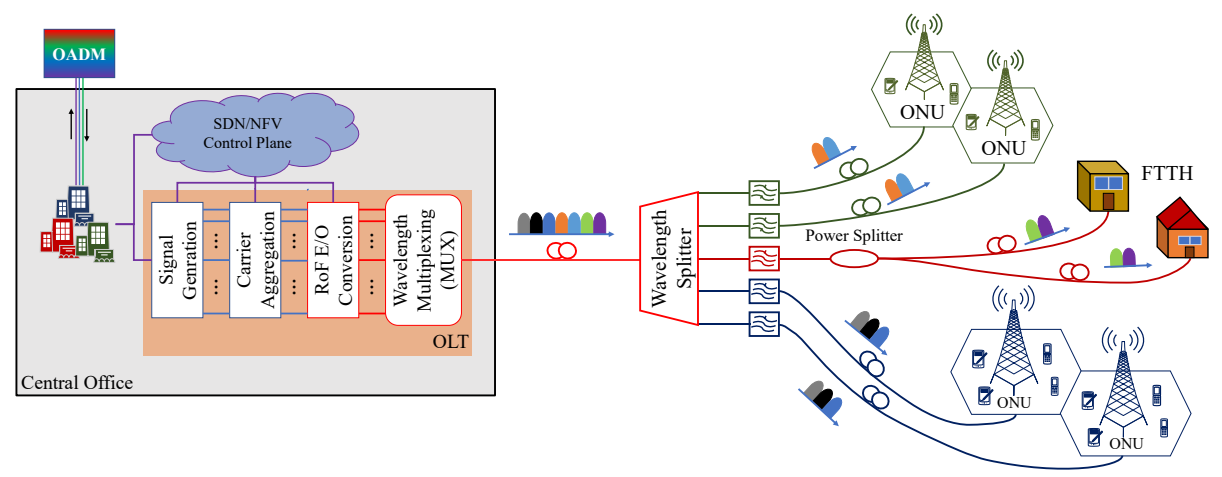

Figure 2: A block diagram of the network proposed by 5G STEP FWD and SILIKA. Optical signals are delivered from the OLT to the ONUs supporting multiple wireless users within a cell or fixed connections to the home.

\subsection{Optical Tone Generation Supported by UDWDM}

The generation and transmission of a radiofrequency (RF) signal is based on optical heterodyning with an ARoF communications scheme [22, 23]. Within the ONUs, two optical signals that have a spacing equal to the carrier frequency of the desired RF electrical signal $\left(\omega_{\mathrm{RF}}=2 \pi f_{\mathrm{RF}}\right)$ beat inside a high-speed photodiode (PD) 24]. For the purposes of 5G STEP FWD, one of the two signals is unmodulated acting as a local oscillator (LO) and the other is modulated based on the format that needs to be transmitted. Both signals need to be coherent preserving the phase information of the RF signals and allowing the transmission of complex modulation formats [25]. These optical waves may be provided by low linewidth lasers driven by an optical phase locked loop or dual-mode lasers [26, 27, 28]. Furthermore, instead of employing multiple laser sources, a feasible and low cost solution is the use of a frequency comb generator in the OLTs transmitting multiple coherent frequency lines [29, 30].

The OLTs are responsible for the transmission of data that needs to be converted from the electrical to the optical domain. This is implemented in the overall structure that is shown in Fig. 3, where multiple laser sources generate light at densely spaced wavelengths. The lasers are tuned by a centralized wavelength locker combined with a high performance thermo-electric cooler pro- 


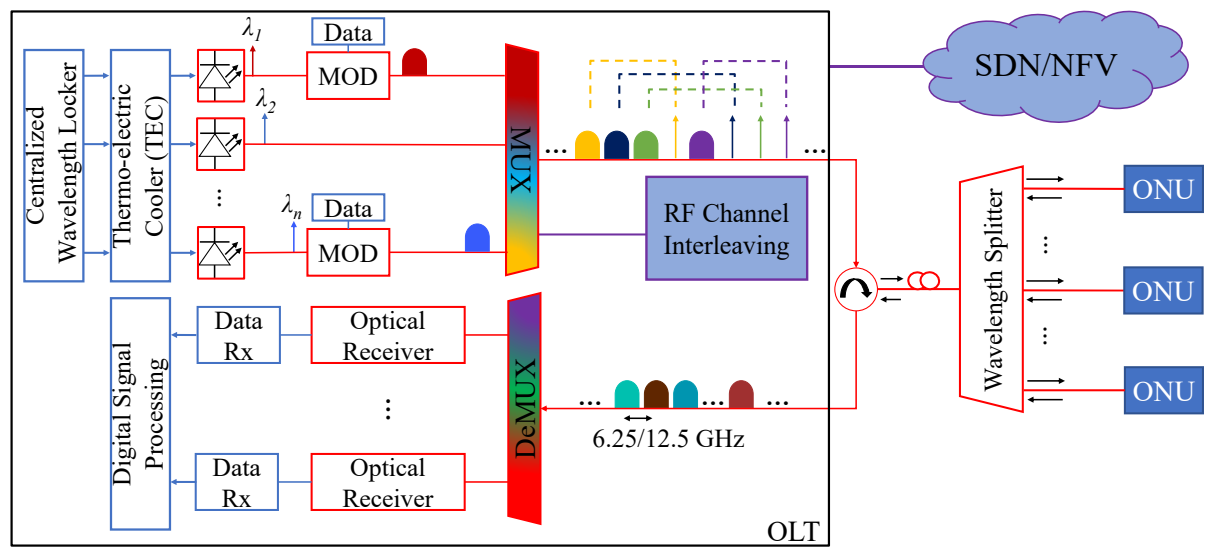

Figure 3: Block diagram illustrating the basic functions of OLTs as well as the optical distribution network; the OLT contains UDWDM signal generation and reception.

hibiting the frequency drift of the sources [31]. Some wavelengths are input to optical modulators where their electrical inputs are the aforementioned data that may be encoded in different modulation formats. The rest of the lasers generate unmodulated optical tones that will function as local oscillators to the mm-wave generation within the ONUs. By assigning UDWDM channel slots and interleaving between modulated and unmodulated tones, flexible selection of the resulting RF frequency is possible, as shown in Fig. 3 . The wavelengths enter an optical multiplexer (MUX) and travel through an optical circulator which allows bidirectionality inside the transmission of the fiber.

The approach using a comb source that can simplify the tone generation is shown in Fig. 4. In that case, an extra demultiplexer (DeMUX) needs to be added into the OLTs since they will be responsible for separating the tones of the comb. The DeMUX may be dependent on external tuning if it is based on active filters such as Fiber Brag Grating (FBG) filters 32 or passive in the case of a photonic integrated array waveguide grating (AWG). In some branches between the DeMUX and MUX, optical modulators are connected and to others the tones propagate (unmodulated) directly to the MUX. It needs to be mentioned that in both approaches the input electrical signals at the optical modulators are not necessarily in baseband. To achieve a more flexible RF frequency selection 


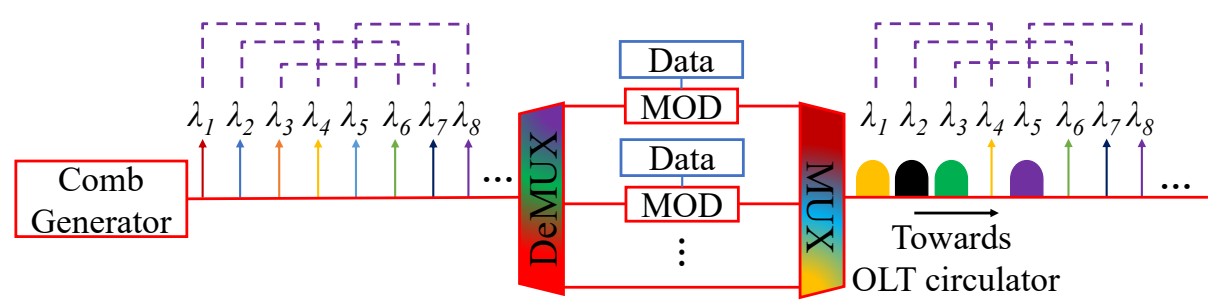

Figure 4: The use of comb generation into the OLTs instead of multiple lasers and the interleaving between modulated and unmodulated tones.

and higher tunability in the case of the comb, signals can be already modulated electrically at an intermediate frequency (IF) based on IF over fiber (IFoF) 33.

The signals at the output of the OLT have a frequency separation that is based according to the ITU-T recommendation G.694.1 [34]. The fixed-grid will evolve to the flex-grid allowing channel slots equal to $12.5 \mathrm{GHz}$ and the next step will lead to a spacing down to $6.25 \mathrm{GHz}$. Therefore, two consequent optical tones would have a frequency separation of either $12.5 \mathrm{GHz}$ or $6.25 \mathrm{GHz}$. Such narrow spacing may increase the probability of channel crosstalk. However, the use of complex modulation formats allows the transmission of multiple bits per symbol increasing significantly the spectral efficiency. Consequently, only a narrow bandwidth fraction of each UDWDM channel is used to ferry the optical signal. Therefore, channel crosstalk between two adjacent channels can be avoided.

At a further step, a UDWDM splitter dictates the separation of wavelengths traveling to a specific ONU. Such a component can be an AWG separating the overall UDWDM signal into 20 or 40 sub-bands. The calculations made in Table 1 summarize some of the important parameters of the proposed UDWDM PON [35, 36, 37. It is assumed that 320 or 640 channels are active and that each ONU is capable of generating four RF signals. Furthermore, some of the tones will be feeding the optical transmitters of the ONUs. In the uplink, the received information from the ONUs travels through the combiner and the circulator. Once more, the received signals will be demultiplexed and then the data will be extracted from an optical heterodyne receiver. Based on the data of the table, 
Table 1: Performance parameters of the presented network concept, where each ONU is capable of generating four RF signals.

\begin{tabular}{lcc}
\hline UDWDM Channel Spacing (GHz) & 12.5 & 6.25 \\
\hline Number of UDWDM Channels & 320 & 640 \\
Power per Channel (dBm)* & -4 & -7 \\
Wavelength Splitting Ratio & $1: 20$ & $1: 40$ \\
Number of Connected ONUs & 20 & 40 \\
Generated RF Signals & 80 & 160 \\
Fiber attenuation (dB/km) & \multicolumn{2}{c}{0.3} \\
Insertion loss per 1:20/1:40 AWG (dB) & \multicolumn{2}{c}{5.5} \\
Insertion loss per UDWDM MUX and DeMUX (dB) & \multicolumn{2}{c}{6} \\
Booster Gain within OLTs (dB) & \multicolumn{2}{c}{$2-6$} \\
Receiver sensitivity (dBm) & -18 \\
\hline *Expected power level after modulation and multiplexing in Fig. 3
\end{tabular}

the optical transmission distance can reach $20 \mathrm{~km}$ which is sufficient for $5 \mathrm{G}$ fronthaul in dense deployments. In the case that reach extenders are available the fiber length may exceed $60 \mathrm{~km}$.

The synthesis of the OLTs are currently cost inefficient and power consuming. This is due to the complexity for the generation and splitting of the UDWDM signals as well as the increased tunability and cooling requirements that such terminal designs demand. In order to carve a roadmap depicting the timescale and financial viability of this scenario the advancements and solutions in photonic integration should be taken into account. Photonic integration may lead to massive production of both multi-tone sources and narrow wavelength selective devices on a compact footprint, scaling down the fabrication costs and allowing this concept to be integrated with existing infrastructures. 


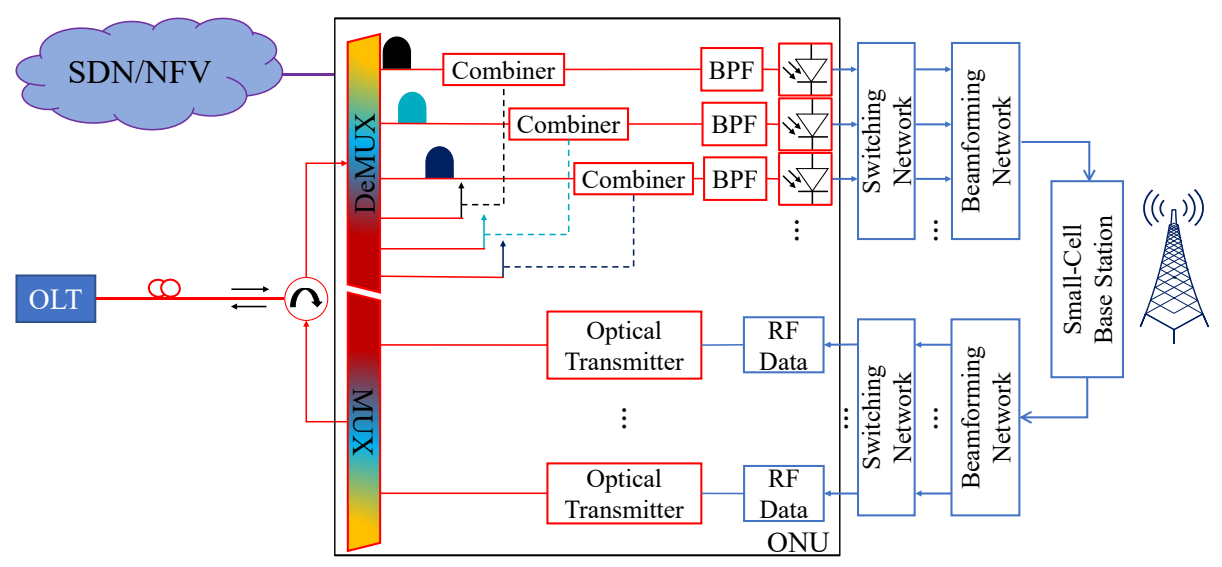

Figure 5: Block diagram illustrating the basic functions of ONUs; the ONU performs conversion between optical and RF domains and connects to the SILIKA beamforming network and antenna.

\subsection{RF Signal Generation for $5 G$}

In the next step, the RF signal is generated in the ONU which is shown in Fig. 5. The modulated optical signals and LOs get separated through a demultiplexer (DeMUX). Channel interleaving regulated by the SDN/NFV plane, allows the proper combination of an optical signal with its corresponding unmodulated tone. The frequency distance between the two wavelengths is equal to a desired RF signal frequency. The two wavelengths go through an optical band pass filter (BPF) which is able to reject the unwanted noise from the desired electrical signal.

After the heterodyne beating at the PD, the output of the ONU can be wirelessly transmitted using the mm-wave antenna provided by SILIKA. There are multiple PDs at the outputs of the ONUs, the number of which depends on the structure of the antenna array and the signal demand inside a cell. Before being transmitted, the RF signals are connected to a switching and beamforming network. The received uplink RF signals are also input to the same network which is connected to optical modulators. With the same process as described in the transmission part of the OLTs, multiple optical signals can be generated based on UDWDM. These signals go through the opposite direction towards the 
OLTs.

\section{Enabling Phased Array Fed Reflector Systems for Mobile Com- munications}

With every step forward in mobile communications, the antenna systems have become more complex. As summarized in [38, in early communications systems antennas were rather simple with an omni-directional radiation pattern in the horizontal plane, which were later replaced by sector antennas and in the latest generation by MIMO antenna systems. For the next step forward the idea of MIMO will be extended by increasing the number of antenna elements to massive MIMO systems [39. This becomes feasible in mobile communications due to the plan to utilize higher frequencies, in particular, mm-waves. The use of massive MIMO has the potential to improve the system capacity and spectral efficiency while keeping the power consumption low [40. One reason for this is that multiple steerable beams can be generated, which provide a strong signal to a certain user while keeping the interference to other users low. Another benefit of massive MIMO is the use of inexpensive and energy-efficient silicon-based amplifiers, which become only applicable if a large amount of antennas are used due to their low output power at mmWaves.

However, besides these advantages there are still challenges that need to be solved. Hardware complexity and signal processing complexity are reaching new dimensions due to the large antenna arrays, which come along with heat dissipation and energy consumption challenges. This makes the realization of such complex antenna systems challenging, as they have to be highly-integrated and energy- and cost-effective at the same time. Therefore, the European projectITN SILIKA proposes an innovative antenna system concept for mobile communications by considering PAF reflectors. PAF reflector systems exhibit a large effective antenna aperture and, therefore, achieve large antenna gain. This allows the reduction of the number of antenna elements as well as the hardware complexity. The major challenge to make PAF reflectors feasible for mobile 


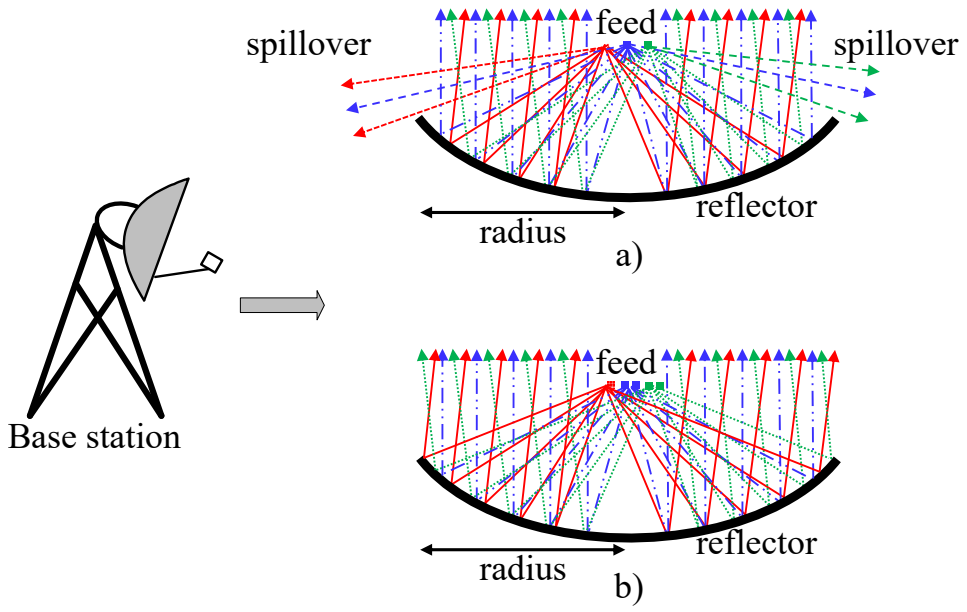

Figure 6: Steering ability of reflector systems by moving the antenna feed away from the focal point where a) shows the problem of spillover if the feed radiation pattern does not illuminate the reflector optimally and b) shows the advantage if the feed radiation pattern can be adjusted by using phase array feeds.

communications, is to mitigate the limited field of view (FoV) of a few degrees.

\subsection{Scanning Ability of Reflector Systems}

In general, to generate off-axis beams for reflector antennas, the feed position of the reflector needs to be changed as shown in Fig. 6. Hence, by using an antenna array as reflector feed, off-axis beams are generated by switching between array elements. This principle is shown in Fig. 6a). The blue element is positioned in the focal point of the reflector, resulting in a broadside beam, the red and green feed elements are displaced from the focal point, generating a scanned beam in a different direction. However, using single active antenna elements (e.g., microstrip patch antennas [41) would result in a low efficiency for example due to spillover as indicated in Fig. 6a). Hence, several elements have to be combined to sub-array feeds. By applying amplitude and phase tapering, a beam in a certain direction with a high efficiency can be achieved, which is studied in [42] and [43] and illustrated in in Fig. 6b). However, off-axis beams with a large scan angle need sub-arrays which has a large off-set from 
the focal point. In case of parabolic reflectors, which focus most of their energy in the focal point, distant sub-arrays for steered beams receive weaker signals, resulting in lower antenna gain for large scan angles. Furthermore, the increased dimension of the array may lead to blockage. The impact of these drawbacks can be mitigated by using optimized reflector and array shapes.

In Fig. 7 the principle of the reflection characteristics of a spherical reflector, in green, a parabolic reflector, in red, and an offset reflector, in black, are illustrated, respectively. One of the most commonly known shapes is the parabolic reflector due to its high antenna gain in broadside direction. As shown by the red arrows in Fig. 7, the reflected rays by a parabola are focused in a focal point. This gives the advantage of a higher achievable gain in comparison to a spherical reflector that has a focal line, marked by green rays in Fig. 7. This higher achievable gain comes along with a narrower scan range in comparison to spherical reflectors. Therefore, it is used in point-to-point applications 44, 45], where high gain and low side lobe levels are needed and a low steering capability can be accepted.

However, if PAF reflectors shall be used in mobile communications to provide service to mobile users, the capability of wide scan angles is indispensable. Spherical reflectors exhibit a larger steering angle that comes along with a lower antenna gain. An example of a spherical reflector with a radius of $60 \mathrm{~cm}$ is given in Fig. 8 for various incidence angles. It can be seen that only a small part of the reflector, marked by a red curve, is efficiently utilized that focuses reflected rays in the area marked by a red box. By changing the angle of incidence the area moves along a curved path that is marked by a green circle in Fig. 8d). This path has a radius, $R_{f}$, of

$$
R_{f}=\frac{R_{s}}{2}
$$

where $R_{s}$ is the spherical reflector radius. Nonetheless, if a feed would be realized along this path, the achieved gain would be degraded as the array would block a large part of the reflector.

A possible solution to the problem of blockage is to use an offset feed 46, 


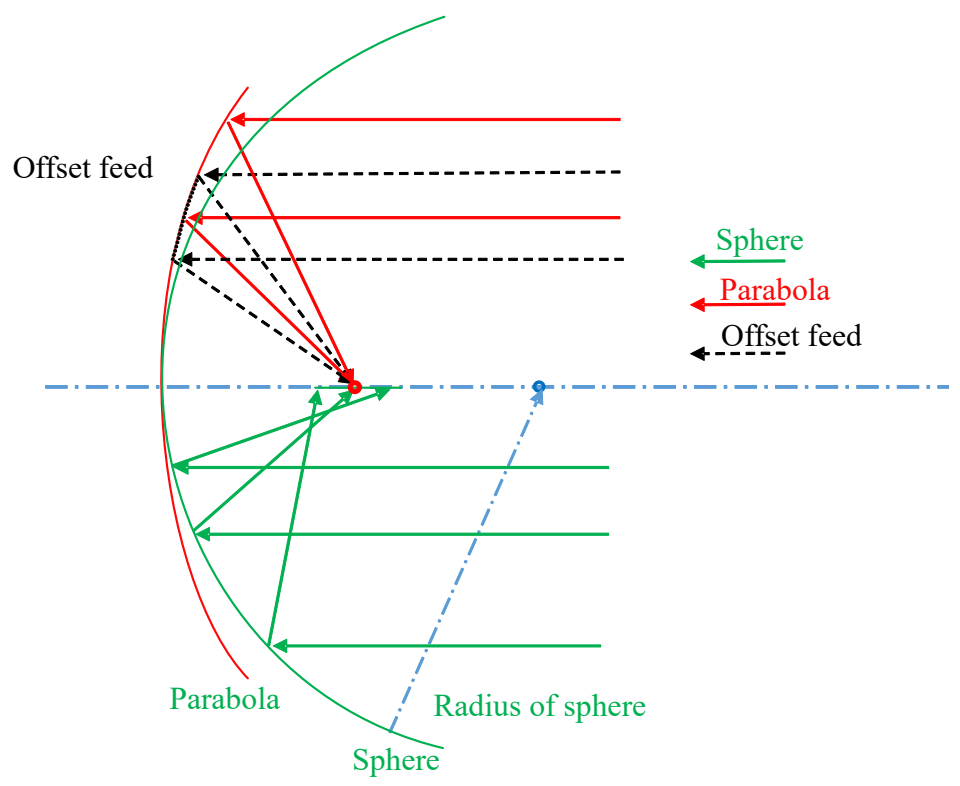

Figure 7: Principle of focusing rays by using spherical, parabolic and off-set fed reflectors.

47. An offset fed reflector can be constructed from a parabolic reflector as shown by the black lines in Fig. 7. This enables designs that allow wide-angle beamsteering without suffering from blockage.

\subsection{SILIKA Approach on the Road to $5 G$}

One of the key aspects of the SILIKA project is to overcome the FoV limitations of PAF reflector systems, in order to enable them for mm-wave $5 \mathrm{G}$ base stations. The needed FoV in azimuth and elevation depends on the considered scenario. Typically, antennas employed for mobile communications cover $\pm 30^{\circ}$ or $\pm 60^{\circ}$ in azimuth [48. Hence, in SILIKA the aim is to achieve an azimuth FoV of $\pm 30^{\circ}$. As shown in Fig. 9, the elevation angle is described by the downtilt angle, $A_{\mathrm{dt}}$, with respect to the horizon. It is calculated by

$$
A_{\mathrm{dt}}=\arctan \left(\frac{h_{\mathrm{BS}}-h_{\mathrm{UE}}}{d}\right),
$$

where $h_{\mathrm{BS}}$ is the base station height, $h_{\mathrm{UE}}$ user equipment height and $d$ is the distance. Therefore, by considering the scenario specifications listed in Table 2 . a $A_{\mathrm{dt}}$ range of $8^{\circ}$ needs to be covered. 


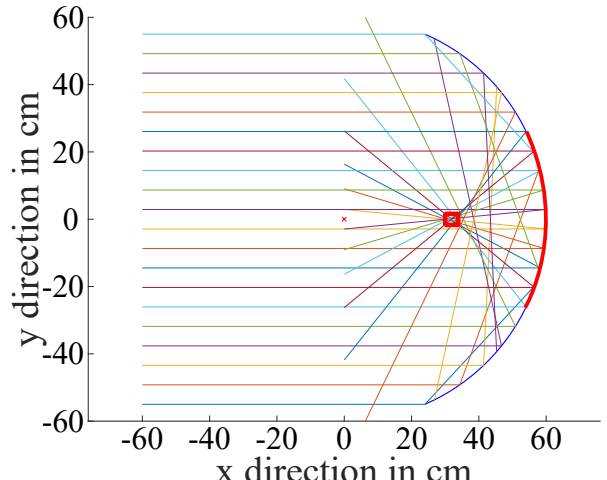

a)

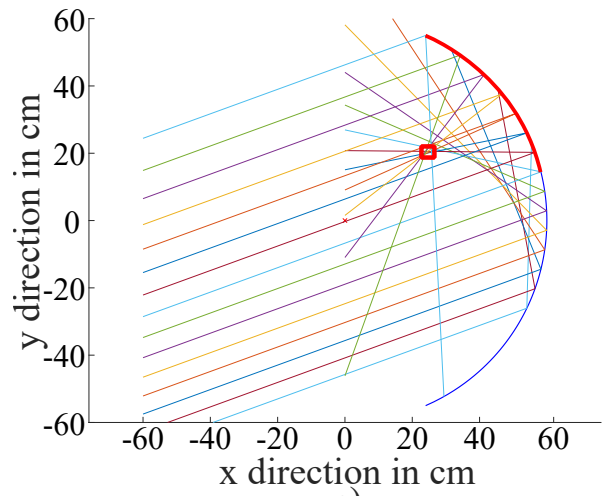

c)

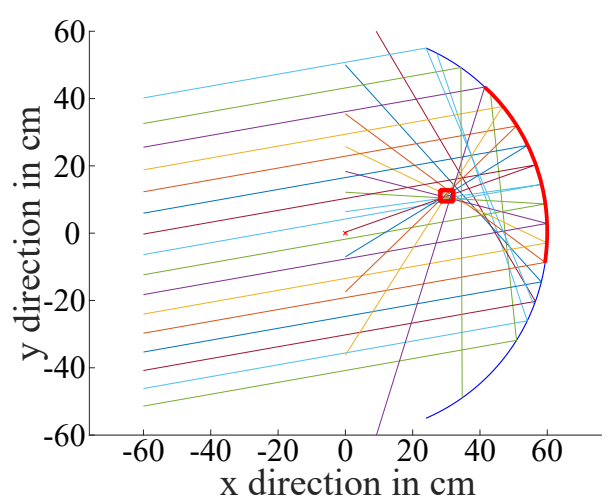

b)

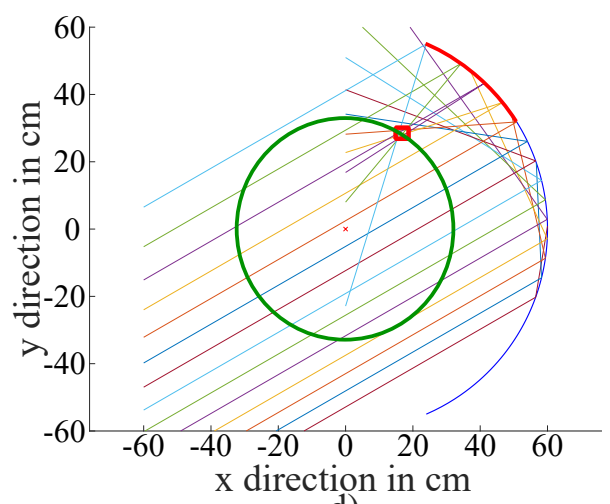

d)

Figure 8: Moving focused area of a spherical reflector at an incident angle a) $0^{\circ}$ b) $-10^{\circ}$ c) $\left.-20^{\circ} \mathrm{d}\right)-30^{\circ}$. Simulated spherical structure has a radius of $60 \mathrm{~cm}$, and a total height of 110 $\mathrm{cm}$. The simulation considers only the first bounce on the reflector of each ray.

Such large FoV ranges can indeed be achieved by PAF reflector systems was already demonstrated in the recently concluded FREEBEAM project conducted at TU Eindhoven [49]. One of the objectives in FREEBEAM was to study and manufacture a broadband wide-scan PAF reflector system for the $20-40 \mathrm{GHz}$ band. The bi-focal double reflector system outcome, [49, shows the ability to achieve more than $40 \mathrm{dBi}$ over a FoV of $\pm 15^{\circ}$ in azimuth at $28.5 \mathrm{GHz}$. However, about 200 array elements are needed to provide full scan range.

Above considerations show that it is possible to provide a wide FoV. However, the number of antenna elements needs to be minimized. Otherwise, in 
Table 2: Resulting number of beams and antenna gains for certain HPBW to cover a given scenario with fixed beams.

\begin{tabular}{|c|c|c|c|c|}
\hline \multicolumn{5}{|c|}{ Scenario considerations } \\
\hline BS height (m) & 15 & & & \\
\hline UE height (m) & 1.5 & & & \\
\hline Distance $(\min ; \max )(\mathrm{m})$ & $75 ; 300$ & & & \\
\hline Azimuth coverage $\left(^{\circ}\right)$ & \pm 30 & & & \\
\hline Downtilt range $\left(^{\circ}\right)$ & 8 & & & \\
\hline \multicolumn{5}{|c|}{ Antenna configurations } \\
\hline $\mathrm{HPBW}\left({ }^{\circ}\right)$ & 1 & 1.5 & 2 & 2.5 \\
\hline Antenna gain (dBi) & 45.3 & 41.8 & 39.3 & 37.3 \\
\hline Number of & 480 & 213 & 120 & 77 \\
\hline beams, $n_{\text {beams }}$ & & & & \\
\hline
\end{tabular}

case of fully digital systems the number of signal paths, as well as the number of digital/analog converters, would be large and similar hardware complexity challenges as for large phased array systems are faced. However, the advantage of PAF reflectors is that only a sub-set of elements contributes to a beam in a certain direction. This attribute makes the use of sub-arrays, which are fed by an analog beamformer $(\mathrm{ABF})$ network, feasible to reduce the number of $\mathrm{RF}$ paths, for instance, by using predefined beams [48, 50]. By using controllable ABF chips, it is possible to combine sub-array signals to enable adaptive beamforming. An example, given in Table 2 shows that it is possible to cover a typical urban cell by using fixed beams with a reasonable number of 77 beams if the half power beamwidth (HPBW) of the reflector is $2.5^{\circ}$. Using the equations from [51] for the relation between antenna gain and HPBW of a parabolic reflector, the antenna gain for this case can be estimated to be approximately $37 \mathrm{dBi}$, which is reasonably high to reach users at a far distance. 


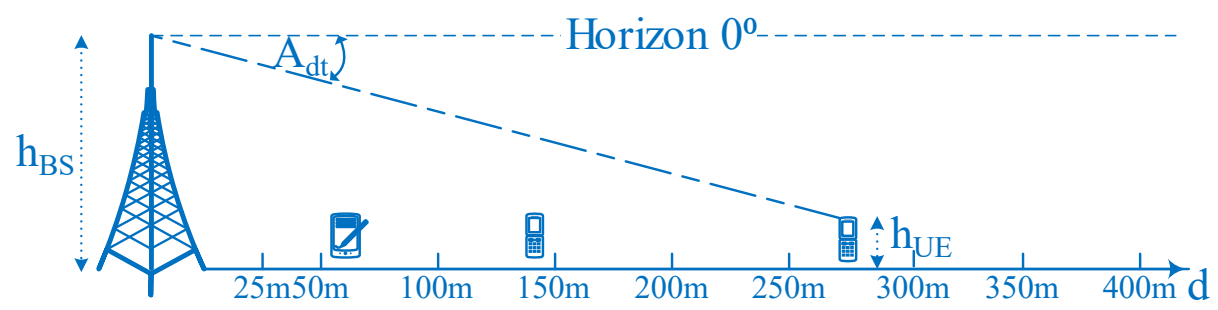

\section{Base station}

Figure 9: Relation between downtilt angle and the geometrical location of base station and user.

\subsection{Connecting Optical Systems to SILIKA via Analog Beamformer Networks}

The interface between the 5G STEP FWD and the SILIKA reflector system is located right after the PD in transmit mode and before the electrical/optical modulator (MOD) in receive mode. Figure 10 shows how the outputs/inputs of the 5G STEP FWD ONU can be connected to the SILIKA PAF reflector.

In transmit mode, the optical system provides multiple PDs, where each provides the signal of a single user. Hence, the number of PDs equals the maximum number of independent beams that can be generated simultaneously. Therefore, it is necessary to be able to provide the signals of each PD to all sub-arrays. To do so, each PD is connected to a series of controllable switches (SW) where the number and constellations depend on the number of sub-arrays and the number of outputs per switch. There are various types of commercially available controllable switches operating in Ka-band. For instance, the Analog Device RF silicon SPxT switches. Each switch in series adds about $1.5 \mathrm{~dB}$ insertion loss, which is compensated by the gain of the ABF chips near the antenna elements. Furthermore, each switch needs supply and digital control voltage that has to be considered in the design. Hence, increasing the flexibility of the board comes along with a higher DC feeding complexity. The accumulated signals of each sub-array are then fed to ABF chips via a circulator, which is used to separate transmit and receive signals. The ABF chips are used to tune amplitude and phase to provide an optimal sampling, which is important to achieve a high antenna efficiency. The number and constellation of the ABF 
network depends on the final reflector design as well as on the sub-array size.

The advantage of this feeding network is that accurate field sampling can be provided by a small ABF network while the signals are distributed via a more inexpensive SW network. This makes it also possible to generate multiple beams for each user to obtain spatial multiplexing gain if due to multi-path phenomena multiple directions are possible. On the other hand, in receive mode all signals can be received simultaneously and are highly separated due to the use of subarrays.

\section{Preliminary Results and Concepts for Future Experiments}

One step towards the first results leading to the merge between 5G STEP FWD and SILIKA is an experimental RoF system [52, 53]. Carrier aggregated 5G OFDM signals with multiple bands were transmitted. In the photonic upconversion scheme that is built based on Fig. 11, the LO and the signal are generated by the same laser source. The laser at the transmitting unit (TU) emits at a carrier frequency $f_{c}$ and the light undergoes carrier suppressed amplitude modulation by a sinusoid coming from a vector signal generator (VSG) at half of the desired RF frequency $f_{R F}$.

The two tones are separated by a wavelength selective switch (WSS). The WSS is a Finisar DWP-EB-AA series with a minimum channel spacing of $50 \mathrm{GHz}$ (DWDM). Therefore, in order to suppress the carrier and achieve the two tone separation $(28 \mathrm{GHz})$, the wavelength of the laser source has to be tuned so that it coincides with the boundaries of two consecutive DWDM channels. As discussed before, a photonic integrated demultiplexer based on AWGs or FBG filters should be better in perfomance and effectiveness than a WSS.

At the output of the WSS, one of the tones is modulated by an electrical signal with the second optical modulator (Mach-Zehnder) and recombined with the LO. The optical power of the LO should be equal to the one of the modulated

optical signal. Consequently, a variable optical attenuator (VOA) is needed in order to achieve this power equality. The signal and the LO are recombined and 


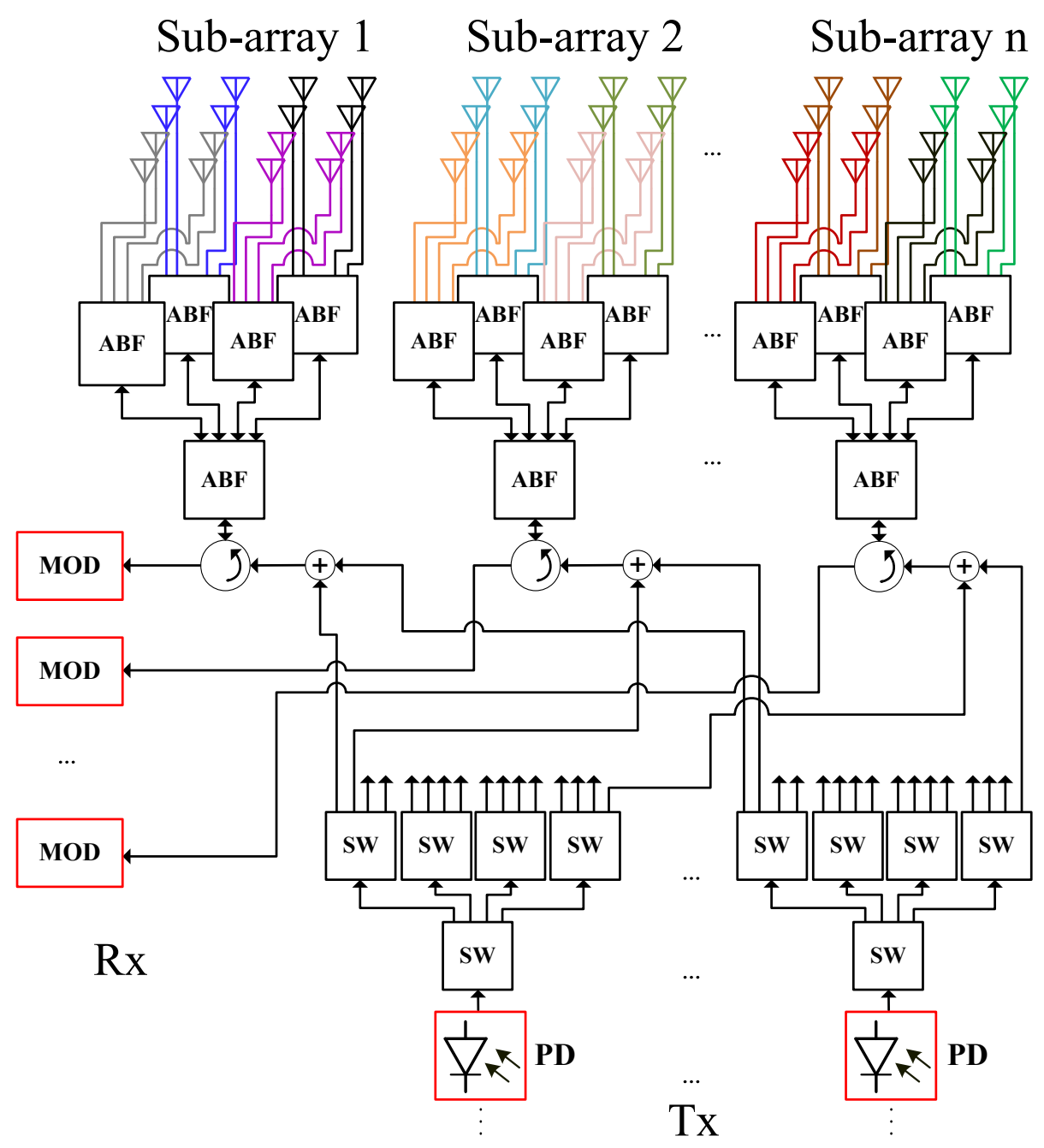

Figure 10: PAF reflector system with a sub-array configuration connected to the 5G STEP FWD interface via switches (SW) and analog beamformer (ABF) chips.

amplified by an optical amplifier. The total signal is introduced to the optical fiber and propagates towards the remote unit (RU), shown in Fig. 12 .

The optical signal arriving at the RU beats inside the PD generating an $\mathrm{RF}$ signal that has a frequency at $28 \mathrm{GHz}$ (within Ka-band). The electrical signal is amplified by a medium power amplifier (MPA). Finally, a pair of horn antennas with $20 \mathrm{dBi}$ gain each is used at the transmitter and receiver. The 


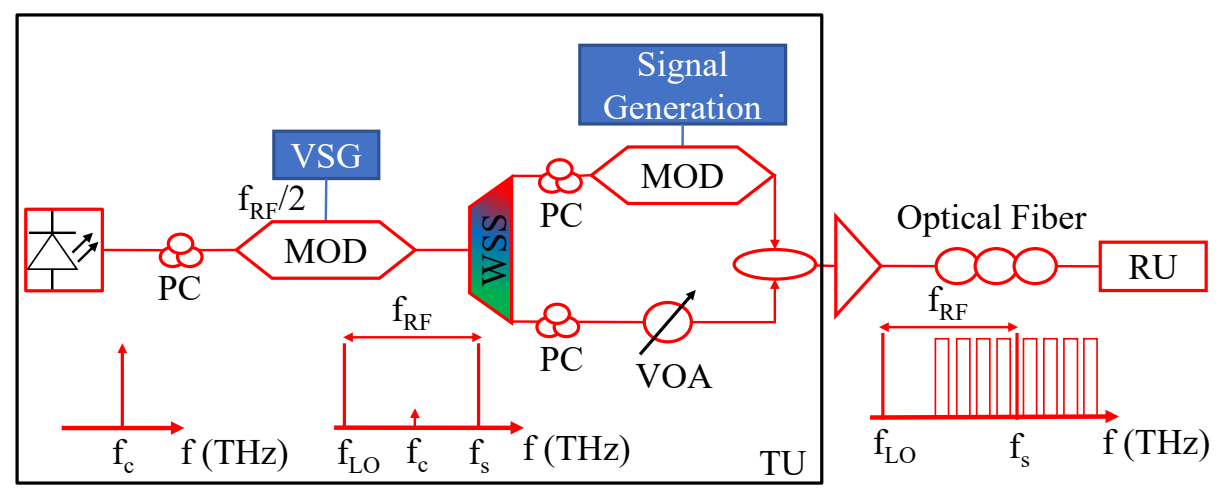

Figure 11: The principles of an experimental OLT, where the optical LO and the signal to be transmitted are generated.

wireless transmission distance is $2.2 \mathrm{~m}$. At the receiver, depicted in Fig. 12 , the RF signal is amplified by a low noise amplifier (LNA) after the antenna and downconverted to a lower frequency by an electrical mixer. Finally, the signal is processed offline by digital signal processing (DSP).

The electrical multiband OFDM signal connected with the optical modulator is synthesized in MATLAB [54, 55] and is based on the suggested numerology and OFDM configuration for 5G [56. The signal is generated by mapping MQAM symbols from a random bit stream onto the OFDM subcarriers spaced at $60 \mathrm{kHz}$. The total number of subcarriers per OFDM symbol is either 2048 or 4096 with 1650 or 3300 being active respectively. Pilot tones are inserted on every $12^{\text {th }}$ subcarrier. Next, the conversion to time domain takes place by using an inverse fast Fourier transform (IFFT). Moreover, a cyclic prefix (CP) with a size of $6.25 \%$ of the OFDM symbol-length is prepended to each OFDM symbol to prevent inter-symbol interference. OFDM frames are formed of 14 OFDM symbols each. Every frame is appended to a preamble equal to two times the OFDM symbol-length. In the experiment, $\mathrm{M}$ is equal to 16 and the number of bands is 8. At the receiver side, the received pilot tones are compared to the transmitted ones, to reverse the impact of the channel. The symbols are demodulated for measuring BER and evaluating the ARoF link.

Figure 13 shows BER with respect to power for 16 QAM symbols and 8 bands 


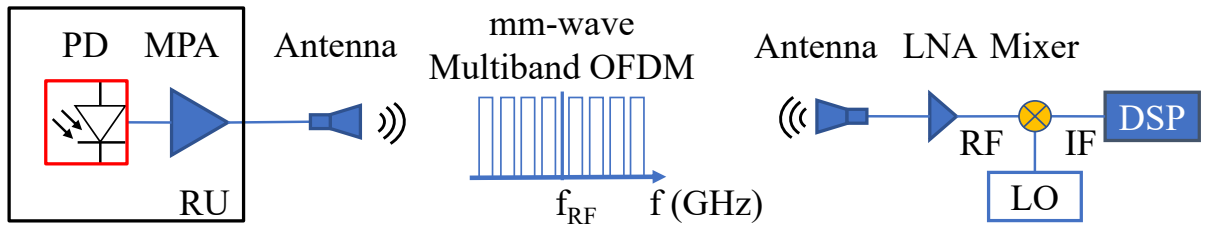

Figure 12: The generation of an RF signal within the RU including a PD and RF components (amplifier and antenna) and the receiver for the wireless transmission of multiband OFDM signals in Ka-band.

as well as the BER limit corresponding to forward error corrections (FEC) with $25 \%$ overhead $(\mathrm{OH})$. It also depicts the received constellation diagrams for all bands. If the transmitter power is increased then an improved BER performance is expected as well as the increase of transmission distances. For the signal with 8 bands in Fig. 13 an average BER below the limit for $25 \%$ overhead FEC is achieved, allowing a data rate of $4.8 \mathrm{Gbit} / \mathrm{s}$ with a bandwidth of $1.96 \mathrm{GHz}$.

The obtained results show a high potential with respect to capacity and data rate. By boosting the power of the transmitted signal, or optimizing even further the system in terms of efficiency of RF signal generation, it is expected that OFDM signals with a greater number of bands and higher order modulation formats will be supported. Finally, these improvements will contribute to the synthesis of the complex system proposed in the concept of this paper.

\section{Conclusion}

This manuscript presents the joint concept of two European projects showcasing an innovative solution for optical-wireless $5 \mathrm{G}$ networks that provide high speed connections with ultra-low latency to a large amount of users.

5G STEP FWD proposes a novel 5G architecture for the backhaul optical network that is based on UDWDM. A large number of OLTs is interconnected through a ring architecture allowing them to communicate, exchange information and provide access to ultra dense mm-wave networks. Within an OLT, multiple optical signals are generated and spaced based on UDWDM standards 


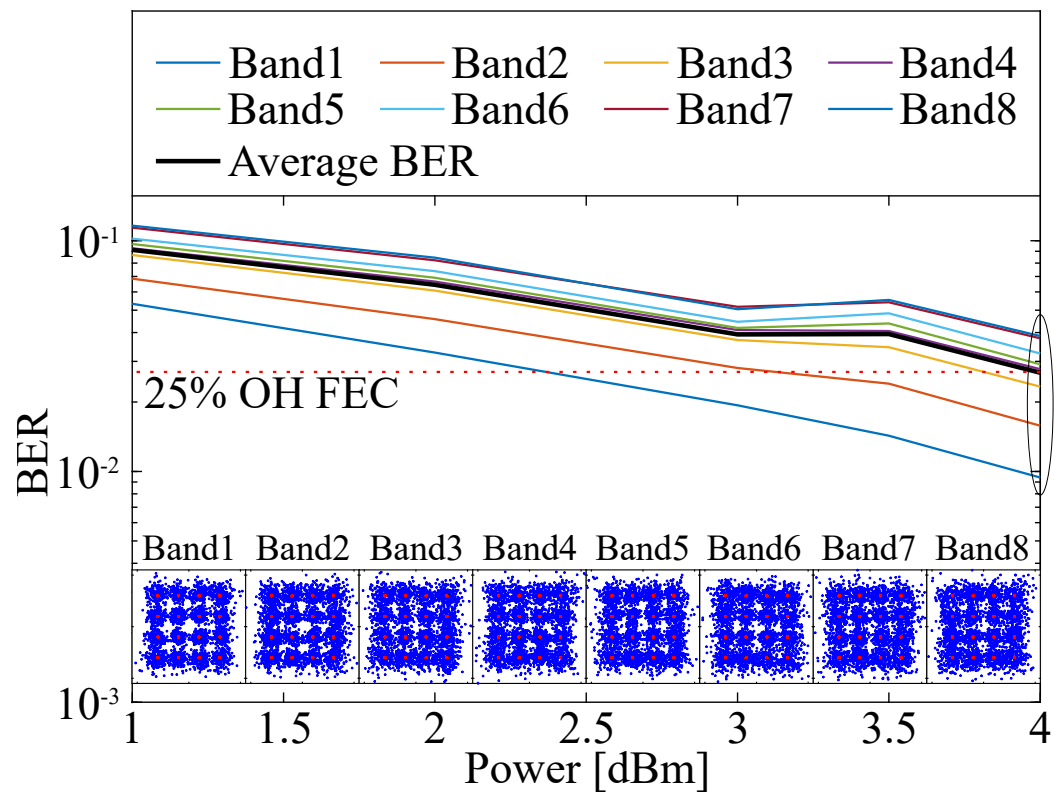

Figure 13: BER as a function of optical power for an 8 band OFDM signal using 16 QAM symbols as a function of the input power at the PD; the constellation diagrams are depicted for optical powers of $4 \mathrm{dBm}$.

and according to the required RF frequencies. Optical heterodyning converts the ARoF to RF signals for wireless transmission.

The outputs of the ONUs are fed to a routing network consisting of controllable switches, which is connected to the sub-array structure via a beamforming network. Due to the routing the actual scanned beam direction is determined while the beamforming network is used to tune amplitude and phase for an optimal field sampling of the PAF reflector. This PAF reflector system is capable of generating multiple beams and independently tracking moving users within a cell by enabling sub-arrays of the antenna leading to focused beams that reach distant users with high antenna gain. This novel approach for mobile communications systems is studied and developed in the course of the EU project-ITN SILIKA.

The first experimental results are shown including the transmission of multiband OFDM signals based on 5G numerology over a $28 \mathrm{GHz}$ ARoF fronthaul 
link. Bit error rates below the limit for a $25 \%$ overhead FEC after wireless transmission over $2.2 \mathrm{~m}$ are achieved. The maximum bit rate is equal to $4.8 \mathrm{Gbit} / \mathrm{s}$ over a bandwidth of $1.96 \mathrm{GHz}$. The obtained results provide important input to the realization of the convergence between 5G STEP FWD and SILIKA.

\section{Acknowledgements}

This work was partially funded by the 5G STEP FWD, SILIKA, and blueS-

PACE projects with funding from the European Union's Horizon 2020 research and innovation programme under grant agreement numbers 722429, 721732, and 762055 , respectively.

\section{References}

[1] W. H. Chin, Z. Fan, R. Haines, Emerging technologies and research challenges for 5G wireless networks, IEEE Wireless Communications 21 (2) (2014) 106-112. doi:10.1109/MWC.2014.6812298.

[2] International Telecommunication Union (ITU) - Telecommunication Standardization Sector, Geneva, FG IMT-2020: Report on Standards Gap Analysis (2016).

[3] B. Ponder, S. Johnston, L. Chodosh, Ericsson Technology Review on Cloud Robotics: 5G Paves the Way for Mass-Market Automation, Charting the future of innovation 93 (2016) 1-14.

[4] The 5G Infrastructure Public Private Partnership (5G-PPP)] [online].

[5] C. Jeong, J. Park, H. Yu, Random access in millimeter-wave beamforming cellular networks: issues and approaches, IEEE Communications Magazine 53 (1) (2015) 180-185. doi:10.1109/MCOM. 2015.7010532.

[6] J. Lee, E. Tejedor, K. Ranta-aho, H. Wang, K. Lee, E. Semaan, E. Mohyeldin, J. Song, C. Bergljung, S. Jung, Spectrum for 5G: Global Status, 
Challenges, and Enabling Technologies, IEEE Communications Magazine 56 (3) (2018) 12-18.

[7] J. Sung, C. Chow, C. Yeh, Y. Liu, G. Chang, Cost-Effective Mobile Backhaul Network Using Existing ODN of PONs for the 5G Wireless Systems, IEEE Photonics Journal 7 (6) (2015) 1-6. doi:10.1109/JPHOT.2015. 2497222

[8] A. H. Jafari, D. López-Pérez, H. Song, H. Claussen, L. Ho, J. Zhang, Small cell backhaul: challenges and prospective solutions, EURASIP Journal on Wireless Communications and Networking 2015 (1) (2015) 206. doi:10. $1186 / \mathrm{s} 13638-015-0426-\mathrm{y}$.

[9] M. Fujiwara, K. Suzuki, N. Yoshimoto, M. Oguma, S. Soma, Increasing splitting ratio of $10 \mathrm{~Gb} / \mathrm{s}$-class PONs by using FW-DMF that acts as low loss splitter for upstream and conventional splitter for downstream, in: OFC 2014, 2014, pp. 1-3.

[10] European Project ITN: 5G System Technological Enhancements Provided by Fiber Wireless Deployments (5G STEP FWD) [online].

[11] Z. Dong, X. Li, J. Yu, Z. Cao, N. Chi, $8 \times 9.95-G b / s$ Ultra-Dense WDMPON on a $12.5-\mathrm{GHz}$ Grid With Digital Pre-Equalization, IEEE Photonics Technology Letters 25 (2) (2013) 194-197. doi:10.1109/LPT.2012. 2233467 .

[12] J. S. Vardakas, I. Tafur Monroy, L. Wosinska, G. Agapiou, R. Brenot, N. Pleros, C. Verikoukis, Towards high capacity and low latency backhauling in 5G: The 5G STEP-FWD vision, in: 19th International Conf. on Transparent Optical Networks (ICTON), 2017, pp. 1-4. doi:10.1109/ ICTON.2017.8025130

[13] Qualcomm Technologies, San Diego, CA, USA, Making 5G NR a reality. Leading the technology inventions for a unified, more capable $5 \mathrm{G}$ air interface (2016). 
[14] European Project ITN: Silicon-based Ka-band massive MIMO antenna systems for new telecommunication services (SILIKA) [online].

[15] M. Arts, M. Ivashina, O. Iupikov, L. Bakker, R. van den Brink, Design of a low-loss low-noise tapered slot phased array feed for reflector antennas, in: Proceedings of the Fourth European Conference on Antennas and Propagation, 2010, pp. 1-5.

[16] M. Cooley, Phased Array Fed Reflector (PAFR) antenna architectures for space-based sensors, in: 2015 IEEE Aerospace Conference, 2015, pp. 1-11. doi:10.1109/AERO.2015.7118963.

[17] J. S. Vardakas, E. Kartsakli, S. Papaioannou, G. Kalfas, N. Pleros, A. Antonopoulos, C. Verikoukis, Quality of Service Provisioning in HighCapacity 5G Fronthaul/Backhaul Networks, in: M. E. Auer, T. Tsiatsos (Eds.), Interactive Mobile Communication Technologies and Learning, Springer International Publishing, Cham, 2018, pp. 797-804.

[18] P. E. Green, Fiber to the home: the next big broadband thing, IEEE Communications Magazine 42 (9) (2004) 100-106. doi:10.1109/MCOM. 2004.1336726

[19] T. Muciaccia, V. Passaro, Future Scenarios for Software-Defined Metro and Access Networks and Software-Defined Photonics, Photonics 4 (2017) 1. doi:10.3390/photonics4010001.

[20] blueSPACE Consortium, EU, Space Division Multiplexing 5G Fronthaul with Analog and Digital Radio-over-Fiber and Optical Beamforming the blueSPACE Concept (2018). doi:10.5281/zenodo.1403140.

[21] S. Rommel, D. Perez-Galacho, J. M. Fabrega, R. Munoz, S. Sales, I. Tafur Monroy, High-Capacity 5G Fronthaul Networks Based on Optical Space Division Multiplexing, IEEE Trans. Broadcasting 65 (2) (2019) 434-443. doi:10.1109/TBC.2019.2901412. 
[22] L. Chorchos, S. Rommel, J. P. Turkiewicz, I. Tafur Monroy, J. J. Vegas Olmos, Reconfigurable Radio Access Unit for DWDM to W-Band Wireless Conversion, IEEE Photonics Technology Letters 29 (6) (2017) 489-492. doi:10.1109/LPT.2017.2656894.

[23] S. Rodrguez, S. Rommel, J. J. Vegas Olmos, I. Tafur Monroy, Reconfigurable radio access unit to dynamically distribute W-band signals in $5 \mathrm{G}$ wireless access networks, Optical Switching and Networking 24 (2017) 21 - 24. doi:10.1016/j.osn.2016.10.002.

[24] J. Yao, Microwave Photonics, Journal of Lightwave Technology 27 (3) (2009) 314-335. doi:10.1109/JLT .2008.2009551

[25] W. Jiang, C. Lin, A. Ng'oma, P. Shih, J. Chen, M. Sauer, F. Annunziata, S. Chi, Simple 14-Gb/s Short-Range Radio-Over-Fiber System Employing a Single-Electrode MZM for 60-GHz Wireless Applications, Journal of Lightwave Technology 28 (16) (2010) 2238-2246. doi:10.1109/JLT. 2010. 2045341 .

[26] P. Brochard, S. Bilicki, A. Shehzad, S. Schilt, T. Sudmeyer, Laser linewidth optimization in a feedback loop, in: 2017 European Conference on Lasers and Electro-Optics and European Quantum Electronics Conference, 2017, pp. $4-3$.

[27] C. F. Wu, X. S. Yan, J. Q. Huang, J. W. Zhang, L. J. Wang, Phase noise reduction by optical phase-locked loop for a coherent bichromatic laser based on the injection-locking technique, Review of Scientific Instruments 89 (1) (2018) 013103. doi:10.1063/1.4993262

[28] H. Weng, Y. Yang, J. Wu, Y. Hao, M. Tang, J. Xiao, Y. Huang, Dualmode microcavity semiconductor lasers, IEEE Journal of Selected Topics in Quantum Electronics 25 (6) (2019) 1-8. doi:10.1109/JSTQE.2019. 2918165. 
[29] V. Ataie, E. Temprana, L. Liu, E. Myslivets, B. P. P. Kuo, N. Alic, S. Radic, Flex-grid compatible ultra wide frequency comb source for $31.8 \mathrm{tb} / \mathrm{s}$ coherent transmission of 1520 udwdm channels, in: OFC 2014, 2014, pp. 1-3.

[30] A. Morales, D. Konstantinou, S. Rommel, T. R. Raddo, U. Johannsen, C. Okonkwo, I. Tafur Monroy, Bidirectional K-band photonic/wireless link for $5 \mathrm{G}$ communications, in: 44th International Conference on Infrared, Millimeter, and Terahertz Waves (IRMMW-THz 2019), 2019, pp. 1-2, in press.

[31] S. Pachnicke, J. Zhu, M. Lawin, A. Wonfor, M. Eiselt, R. Cush, R. Turner, P. Firth, M. Wale, R. V. Penty, I. H. White, J. Elbers, Novel WDM-PON System with Shared Wavelength Locking and Full C-Band Tunability, in: Photonic Networks; 15. ITG Symposium, 2014, pp. 1-5.

[32] M. Ferchichi, M. Najjar, H. Rezig, Design of temperature-strain tunable udwdm, dwdm, wdm fbg filter for passive optical network access, in: 2008 2nd ICTON Mediterranean Winter, 2008, pp. 1-5. doi:10.1109/ICTONMW. 2008.4773074

[33] M. Sung, S. Cho, K. S. Kim, H. Kwon, B. Kang, D. S. Oh, D. Lyu, H. Lee, S. M. Kim, J. H. Lee, H. S. Chung, Demonstration of IFoF based 5G mobile fronthaul in $28 \mathrm{GHz}$ millimeter wave testbed supporting giga-bit mobile services, in: 2017 Optical Fiber Communications Conference and Exhibition (OFC), 2017, pp. 1-3.

[34] ITU-T Recommendation G.694.1, Spectral grids for WDM applications: DWDM frequency grid (February 2012).

[35] K. Grobe, M. Roppelt, M. Eiselt, J. Elbers, Combined reach, client-count, power-consumption, and cost analysis of wdm-based next-generation pon, in: 2011 37th European Conference and Exhibition on Optical Communication, 2011, pp. 1-3. 
[36] M. Forzati, A. Bianchi, J. Chen, K. Grobe, B. Lannoo, C. Mas Machuca, J. Point, B. Skubic, S. Verbrugge, E. Weis, L. Wosinska, D. Breuer, Nextgeneration optical access seamless evolution: concluding results of the European FP7 Project OASE, IEEE/OSA Journal of Optical Communications and Networking 7 (2) (2015) 109-123. doi:10.1364/JOCN.7.000109.

[37] K. Grobe, M. Eiselt, Wavelength Division Multiplexing: A Practical Engineering Guide, Wiley, 2014, Ch. 6, pp. 267-340. doi:10.1002/ 9781118755068.ch06

[38] C. Beckman, B. Lindmark, The Evolution of Base Station Antennas for Mobile Communications, in: 2007 International Conference on Electromagnetics in Advanced Applications, 2007, pp. 85-92. doi:10.1109/ICEAA. 2007.4387244

[39] S. Parkvall, E. Dahlman, A. Furuskar, M. Frenne, NR: The New 5G Radio Access Technology, IEEE Communications Standards Magazine 1 (4) (2017) 24-30. doi:10.1109/MCOMSTD . 2017.1700042.

[40] N. H. M. Adnan, I. M. Rafiqul, A. H. M. Z. Alam, Massive MIMO for Fifth Generation (5G): Opportunities and Challenges, in: 2016 International Conference on Computer and Communication Engineering (ICCCE), 2016, pp. 47-52. doi:10.1109/ICCCE.2016.23.

[41] A. B. Smolders, R. M. C. Mestrom, A. C. F. Reniers, M. Geurts, A Shared Aperture Dual-Frequency Circularly Polarized Microstrip Array Antenna, IEEE Antennas and Wireless Propagation Letters 12 (2013) 120-123. doi: 10.1109/LAWP.2013.2242427

[42] A. Dubok, A. Al-Rawi, N. Tessema, E. Tangdiongga, M. H. A. J. Herben, G. Gerini, A. B. Smolders, Double-Reflector Configuration for Optimal Exposure of Wideband Focal-Plane Arrays With Optical Beamforming, IEEE Transactions on Antennas and Propagation 65 (8) (2017) 4316-4321. doi:10.1109/TAP.2017.2709620. 
[43] M. V. Ivashina, O. Iupikov, R. Maaskant, W. A. van Cappellen, T. Oosterloo, An Optimal Beamforming Strategy for Wide-Field Surveys With Phased-Array-Fed Reflector Antennas, IEEE Transactions on Antennas and Propagation 59 (6) (2011) 1864-1875. doi:10.1109/TAP.2011. 2123865

[44] M. Nagasaka, S. Nakazawa, S. Tanaka, Prototype of a dual-circularly polarized parabolic reflector antenna with microstrip antenna array for 12ghz band satellite broadcasting reception, in: 2016 10th European Conference on Antennas and Propagation (EuCAP), 2016, pp. 1-5. doi: 10.1109/EuCAP. 2016.7481691.

[45] P. Harrop, J. Magarshack, R. Dessert, J. R. Forrest, Satellite communications II: Television for everyone: Low-cost Earth stations that can select from a broad menu of satellite broadcasts are sought, IEEE Spectrum 17 (3) (1980) 54-56. doi:10.1109/MSPEC.1980.6330327.

[46] A. W. Rudge, N. A. Adatia, Offset-parabolic-reflector antennas: A review, Proceedings of the IEEE 66 (12) (1978) 1592-1618. doi:10.1109/PROC. 1978.11170.

[47] S. K. Rao, Advanced Antenna Technologies for Satellite Communications Payloads, IEEE Transactions on Antennas and Propagation 63 (4) (2015) 1205-1217. doi:10.1109/TAP.2015.2391283.

[48] A. Elsakka, T. A. H. Bressner, A. J. van den Biggelaar, A. Al-Rawi, U. Johannsen, M. V. Ivashina, A. B. Smolders, On the use of focal-plane arrays in mm-wave 5G base stations, in: 12th European Conference on Antennas and Propagation (EuCAP 2018), Institution of Engineering and Technology (IET), United Kingdom, 2018, pp. 1-4. doi:10.1049/cp.2018.1207.

[49] A. B. Smolders, A. Dubok, N. M. Tessema, Z. Chen, A. N. H. Al-Rawi, U. Johannsen, T. A. H. Bressner, D. Milosevic, H. Gao, E. Tangdiongga, G. Gerini, P. G. M. Baltus, M. Geurts, A. M. J. Koonen, Building 5G 
millimeter-wave wireless infrastructure: wide-scan focal plane arrays with broadband optical beamforming, IEEE Antennas and Propagation Magazine.

[50] T. A. H. Bressner, U. Johannsen, A. B. Smolders, Single shot DoA estimation for large-array base station systems in multi-user environments, in: Loughborough Antennas Propagation Conference (LAPC 2017), 2017, pp. 1-4. doi:10.1049/cp.2017.0288.

[51] D. Minoli, Satellite Systems Engineering in an IPv6 Environment, CRC Press, 2009.

[52] S. Rommel, L. C. P. Cavalcante, A. G. Quintero, A. K. Mishra, J. J. Vegas Olmos, I. Tafur Monroy, W-band photonic-wireless link with a Schottky diode envelope detector and bend insensitive fiber, Opt. Express 24 (11) (2016) 11312-11322. doi:10.1364/OE.24.011312.

[53] D. Konstantinou, A. Morales, S. Rommel, T. R. Raddo, U. Johannsen, I. Tafur Monroy, Analog radio over fiber fronthaul for high bandwidth $5 \mathrm{~g}$ millimeter-wave carrier aggregated ofdm, in: 21st International Conference on Transparent Optical Networks, 2019, pp. 1-4, in press.

[54] Y. S. Cho, J. Kim, W. Y. Yang, C. G. Kang, MIMO-OFDM Wireless Communications with MATLAB, Wiley Publishing, 2010.

[55] L. C. P. Cavalcante, S. Rommel, R. Dinis, L. G. Q. Silveira Junior, L. F. Q. Silveira, I. Tafur Monroy, Performance Evaluation of WaveletCoded OFDM on a $4.9 \mathrm{~Gb} / \mathrm{s}$ W-Band Radio-Over-Fiber Link, J. Lightwave Technol. 35 (14) (2017) 2803-2809. doi:10.1109/JLT.2017.2701358.

[56] Nokia, Alcatel-Lucent Shanghai Bell, Resource block and guard band arrangement supporting mixed numerology, R1-167260, 3GPP TSG RAN WG1 \#86 (2016). 